\title{
A Therapeutic Target for Hormone-independent Estrogen Receptor-positive Breast Cancers
}

\author{
Debajit K. Biswas, ${ }^{1}$ Antonio Cruz, ${ }^{1}$ Nicole Pettit, ${ }^{1}$ George L. Mutter $^{2}$ and Arthur B. Pardee ${ }^{1}$ \\ ${ }^{1}$ Division of Cancer Biology, Dana-Farber Cancer Institute, and \\ ${ }^{2}$ Department of Pathology, Brigham and Women's Hospital, Harvard Medical School, Boston, \\ Massachusetts, U.S.A. \\ Communicated by AB Pardee. Accepted September 15, 2000.
}

\begin{abstract}
Background: The action of the steroid hormone estradiol (E2) is mediated via interaction with a specific receptor (ER) that initiates a series of events downstream, leading to the modulation of hormone-responsive genes and cell proliferation. Antihormones also bind, but do not confer the active configuration to ER, thereby, blocking the transmission of E2-ER-initiated signals for cell proliferation. Although these compounds qualify for successful therapy of ER-positive [ER (+)] breast cancer patients, only a fraction of patients responds to antihormone treatment. In this study, the functional status of ER is determined to identify alternative targets for therapy of antihormone-resistant ER $(+)$ breast cancers.

Method: The interaction of ER with a specific DNA sequence, designated as E2 response element (ERE), was targeted to assess the functional state of ER. ER-ERE
\end{abstract}

complex formation was measured by electrophoretic mobility shift assay (EMSA) and by a newly developed technique, based on the preferential binding of DNA-protein complex to a nitrocellulose membrane (NMBA) that measures both total and functional fraction of ER.

Results: The NMBA assay identified functional variants of ER among ER $(+)$ breast cancer cell lines and breast tumor biopsy specimens. ER of (21PT) cells did not bind E2 and these cells were tamoxifen (TAM) resistant. However 21PT cells were sensitive to a calmodulin (CaM) antagonist, W7, that blocked ERE-ER complex formation.

Conclusions: ER variants of the 21PT type were detected among breast cancer biopsy specimens, emphasizing the significance of an alternative therapeutic target for TAMresistant ER $(+)$ human breast cancers with compounds such as W7.

\section{Introduction}

Stimulated growth of breast cancer cells by hormones, more specifically estrogen (E2) and progesterone, is the basis of antihormone therapy for breast cancer patients (1-3). This is based on the mode of action of the steroid family of receptors, such as estrogen receptor (ER), which are hormone-activated transcription factors. The role of E2-ER interaction in breast cancer cell proliferation is further elucidated by the expression of human ER in ER-negative [ER (-)] MDA-MB-231 cells, which restores the hormonedependent ER-positive [ER (+)] phenotypes in these cells (4). Conversely, expression of dominant negative ER in ER $(+)$ breast cancer cells suppresses estrogen-stimulated cell proliferation and E2-induced endogenous gene expression (5). Interaction of E2 releases the inhibitory heat shock protein, hsp90, from ER and initiates a series of downstream events (6-8), resulting in modulation of expression of genes

Address correspondence and reprint requests to: Debajit K. Biswas, Division of Cancer Biology, Dana-Farber Cancer Institute,

44 Binney Street, Boston, MA 02115, U.S.A. Phone: 617-632-4684; Fax: 617-632-4680; E-mail: biswas@mbcrr.harvard.edu responsible for enhanced proliferation of breast cancer cells (9-11). Antihormones, such as tamoxifen (TAM) bind to ER, but do not confer the active configuration to this receptor, thereby, blocking subsequent events and acting as antagonists $(2,10,12-14)$. TAM and other compounds, such as raloxifen (RAL), exhibit conformational modification of ER and also can act as agonists in uterine and bone cells $(2,15-17)$. These diverging conformational states of ER induced by compounds in different cell types are believed to be mediated via tissue specific proteinprotein interactions (15).

The therapeutic activity of antihormones should be effective for all ER $(+)$ breast cancers. However, only $60 \%$ of ER (+) breast cancer patients respond to tamoxifen and other antihormone treatments. The remaining ER $(+)$ and all ER $(-)$ breast cancers constitute a substantial fraction that do not respond to antihormone therapy. The signal for enhanced proliferation of ER (-) breast cancer cells is proposed to be initiated by the interaction of epiderrmal growth factor (EGF) with epidermal growth factor receptor (EGFR) and transmitted via a pathway in which active nuclear factor $-\kappa \mathrm{B}(\mathrm{NF}-\kappa \mathrm{B})$ plays an important 
role by regulating the level of the cell cycle regulatory proteins (18). Thus, NF- $\kappa \mathrm{B}$ is a potential target of therapy for ER $(-)$ breast cancer patients (18). The transition from E2-dependent to -independent phenotype can develop nonresponsiveness of some breast cancers to antihormones and has been identified with E2-regulated proteins (19).

The feasibility of antihormone therapy is determined by the level of ER and its functional state. ER and progesterone receptor (PR) levels, the latter being induced by E2-ER-E2 response element (ERE) interaction, are routinely measured in breast cancer biopsy specimens, either by radioactive hormone binding assays or by immunodetection with receptor-specific antibodies. Some ER variants are not readily detected by these assays. To overcome this difficulty and subclassify ER (+) breast cancers, we developed an alternative downstream functional assay that depends upon ER-interaction with its response element, ERE (20). By using this approach, we identified ER variants among established breast cancer cell lines and breast cancer biopsy specimens, which potentially could predict appropriate therapies for different types of breast cancer patients. The receptor in one class of ER $(+)$ breast cancers, such as 21 PT cells, does not bind to E2 $(21,22)$ and is resistant to antihormones (23), but it is sensitive to the calmodulin (CaM)-antagonist, W7 (24). Results presented demonstrate that the downstream event of ER-CaM complex formation might provide an alternative therapeutic target for antihormone-resistant ER $(+)$ breast cancer patients.

\section{Materials and Methods \\ Cell Lines}

The ER (+) MCF-7 and T47D, and ER (-) MDAMB-231 cell lines were obtained from American Type Culture Collection, Rockville, MD (ATCC). The 21PT cell line was established from a primary breast carcinoma by Sager and colleagues $(21,22)$. The 21PT cell line initially was described by these investigators as ER $(-)$, based on ligand binding assay $(21,22)$. Subsequently, ER-specific mRNA, ER protein, and E2-responsive gene product, PR, were detected in 21PT cells, which now is designated as ER (+) (23). These breast cancer cells were maintained in a stock medium [Dulbecco's modified Eagle's medium (DMEM, Gibco BRL Life Technologies, Rockville, MD) supplemented with $10 \%$ fetal bovine serum (FBS, Gemini Bio Products, Woodland, CA) and $2.8 \mu \mathrm{M}$ hydrocortisone, $1 \mu \mathrm{g} / \mathrm{ml}$ insulin, and $12.5 \mathrm{ng} / \mathrm{ml}$ EGF, Sigma Chemicals, St. Louis, MO] at $37^{\circ} \mathrm{C}$ in $5 \% \mathrm{CO}_{2}$ and $95 \%$ air.

\section{Materials}

Monoclonal anti-ER antibody, TEl-11, raised against C-terminal amino acid residues 300-595 of human ER, and monoclonal anti-PR antibody (PgR-Ab-1), raised against purified human proges- terone receptor, were obtained from Neo Markers (Fremont, CA). Purified human recombinant ER was purchased from Pan Vera Corp. (Madison, WI). The Hybond nitrocellulose membrane and enhanced chemiluminescent protein (ECL) immunodetection kit was from Amersham Pharmacia-biotech, UK. Synthetic single-stranded complementary oligonucleotides containing a wild type ERE motif ( $5^{\prime}$ GTCCAAAGTCAGGTCACAGTGACCTGATCAAAGTT 3') and complementary oligonucleotides with progesterone receptor response element (PRE) motif $\left(5^{\prime}\right.$ GGAACAATCAGTTCC 3') were obtained from Integrated DNA Technology, Inc. (Coraville, IA). The single-stranded DNA sequences were annealed to generate the double-stranded ERE or PRE oligonucleotide. 17 $\beta$-Estradiol (E2), hydrocortisone, insulin, dithiothreitol, dimethyl sulfoxide, phenylmethylsulfonyl fluoride, and W7 [N-(6-aminohexyl)5-chloro-1-naphthalene sulfonamide hydrochloride] were obtained from Sigma Chemicals (St. Louis, MO).

\section{Preparation of Cell and Tissue Extracts}

Stock cultures of breast cancer cell lines were grown in DMEM supplemented with FBS and growth factors as described above. Nuclear extracts were prepared as described previously $(20,23-25)$. In brief, the frozen human breast tumor specimens were thawed, minced, and homogenized in buffer II (25). The homogenized suspension was then centrifuged at 800 RPM for $5 \mathrm{~min}$, followed by centrifugation of the low speed supernatant at 10,000 RPM for $10 \mathrm{~min}$ in rotor $\mathrm{J} 18$ in a refrigerated centrifuge (Beckman Instrument, Fullerton, CA). The supernatants then were dialyzed against a large volume of buffer III (25), protein content was determined (26), and saved in small aliquots at $-70^{\circ} \mathrm{C}$, and used for immunodetection assay, electrophoretic mobility shift assay (EMSA), and nitrocellulose membrane binding assay (NMBA).

Immunodetection of total ER and PR was accomplished by our newly developed NMBA protocol (20). An aliquot of the cellular or tissue extracts (10-20 $\mu \mathrm{g}$ protein) was incubated in the presence or absence of nonradioactive ERE-oligonucleotide under conditions for the formation of ER-ERE/Progesterone Receptor-Progesterone Receptor Response Element (PR-PRE) complex $(7,24)$. The reaction mixtures were applied to a nitrocellulose membrane assembled in a multi-slot blot or dot blot system. The samples then were adsorbed to the membrane for $15 \mathrm{~min}$ at room temperature, washed with binding buffer, and reacted with specific primary antibody, followed by secondary antibody conjugated with horseradish peroxidase, and finally, an ECL immunodetection system (Amersham Life Sciences). The immunodetection signals were quantitated by densitometric scanning, followed by integration of the signals with program version 1.0.2. (Molecular Dynamics, Sunnyvale, CA). 
Detection of DNA-bound ${ }^{32} \mathrm{P}$-ERE-ER or ${ }^{32} \mathrm{P}-$ PRE-PR complexes was determined similarly by NMBA (20). ${ }^{32} \mathrm{P}$-end labeled synthetic doublestranded oligonucleotides of ERE or PRE were used, replacing the nonradioactive oligonucleotide. Reactions containing 5-10 $\mu \mathrm{g}$ of nuclear protein from cultured cells or 10-20 $\mu \mathrm{g}$ from tissue extracts of biopsy specimens, plus $1 \mathrm{ng}$ of ${ }^{32} \mathrm{P}$-labeled (approximately 30,000 CPM) of ERE or PRE (shown above), were incubated for $30 \mathrm{~min}$ at room temperature and applied to a prewashed nitrocellulose membrane assembled in the multi-dot blot system, and treated and washed as described above (20). ${ }^{32} \mathrm{P}$-ERE-ER or

${ }^{32}$ P-PRE-PR complex was detected by autoradiography of the membrane, quantitated by densitometric scanning and integration.

The ${ }^{32} \mathrm{P}-\mathrm{ERE}-\mathrm{ER}$ and ${ }^{32} \mathrm{P}$-PRE-PR complexes also were determined by EMSA with nuclear extracts from cultured cells or tissue extracts $(23,24)$. The specificity of this DNA-protein interaction was established by carrying out a reaction with nuclear extract from ER (-) MDA-MB-231 cells in the absence or presence of purified recombinant ER (24). The intensity of this band or dot was quantitated by scanning with a densitometer as described above, and the results were presented as integrated band or dot intensity (arbitrary numbers Fig. 4C).

\section{Results}

Measurement of Total and Functional Fraction of ER and $P R$ by Nitrocellulose Membrane Binding Assay (NMBA)

In this study, we used a newly developed experimental method (NMBA) to determine the functional state of ER by monitoring ERE-ER complex formation, which is an event downstream of E2-ER interaction (20). Both free and DNA-bound ER bind to nitrocellulose membrane. Thus, membrane-bound total ER can be measured by immunodetection with an anti-ER antibody coupled ECL system and ${ }^{32} \mathrm{P}-$ ERE-ER complex can be determined by autoradiography with this assay. The receptor proteins in nuclear extracts from the indicated ER $(+)$ and ER $(-)$ breast cancer cell lines were immobilized on nitrocellulose membrane and immunodetected with antiER antibody (Fig. 1A, left panel). The level of PR, which was an ER-responsive gene product, was determined similarly (Fig. 1A, right panel). ER and PR in nuclear extracts of both the ER $(+)$ 21PT (row 2 ) and T47D (row 3) cells, gave positive signals in this assay. Reactions containing extracts from ER (-) MDA-MB-231 cells (Fig. 1A, row 1) and no extracts in the presence of the same amounts of bovine serum albumin (BSA; data not shown) generated no such signals, establishing the specificity of the positive signals observed with extracts from the ER (+) cells.

The autoradiographic signals on a nitrocellulose membrane obtained from the reactions containing ${ }^{32} \mathrm{P}$-labeled oligonucleotides represented only the immobilized ${ }^{32} \mathrm{P}$-ERE bound to ER (Fig. 1B, left panel) or ${ }^{32}$ P-PRE-PR (Fig. 1B, right panel), representing the functional fraction of the receptors. The very low signal observed with ${ }^{32} \mathrm{P}$-oligonucleotide in the presence of BSA represented the nonspecific binding of the free probe (Fig. 1B, row 1). Nuclear extract of ER (-) MDA-MB-231 cells did not form either ${ }^{32}$ P-ERE-ER (Fig. 1B, row 5, left panel) or ${ }^{32}$ P-PRE-PR (Fig. 1B, row 5, right panel), as indicated by very low nonspecific autoradiographic signals. However, nuclear extracts from ER (+) breast cancer cell lines MCF-7 (Fig. 1B, row 4), T47D (Fig. 1B, row 6), and 21PT (Fig. 1B, row 3) formed ${ }^{32}$ P-ERE-ER (Fig. 1B, left panel) and ${ }^{32}$ P-PRE-PR (Fig. 1B, right panel) complexes. The immunodetection assay (Fig. 1A) gave a qualitatively pattern of signals similar to that obtained with ${ }^{32} \mathrm{P}$-labeled oligonucleotide (Fig. 1B). PR is an E2responsive gene product, and the level of PR, in general, reflected the functional state of ER. Thus, both binding of ER to ${ }^{32} \mathrm{P}$-ERE and detection of PR established the functional state of ER.

Inhibition of ERE-ER Interaction and Growth of ER (+) Breast Cancer Cell Lines in Response to a Calmodulin (CaM) Antagonist W7

Although ER in 21PT cells did not bind to ${ }^{3} \mathrm{H}-\mathrm{E} 2$, ER mRNA and ER protein were detected in these cells $(23,24)$. Thus, 21 PT cells had an ER variant that lost its hormone-binding property. Preliminary DNA sequence analysis of the 1660-2253 bp region of the ligand-binding domain of ER molecule of MCF-7 and 21PT cells did not reveal any significant differences in this coding region between the cell types (data not shown). Possible mutation in the rest of the ligand-binding or DNA-binding domains of ER molecule in 21PT cells is currently under investigation.

The ERE-ER interaction and transactivation of hormone responsive promoters were mediated independent of initial hormone interaction with ER in ER (+) 21PT cells; whereas, these downstream events were dependent on E2 in ER (+) MCF-7 cells (23). Our previous results also revealed that CaM was an essential component of ERE-ER complex formation and transactivation of responsive PS2 promoter (24). The CaM antagonist, W7, inhibited ERE-ER interaction in both of these ER $(+)$ cell lines, as measured by NMBA (Fig. 2A) and EMSA (Fig. 2B). Densitometric quantitation of the signals demonstrated $63-77 \%$ inhibition of ERE-ER complex formation by $25 \mu \mathrm{M}$ W7 in 21PT cells, and similar treatment of MCF-7 cells consistently showed a somewhat lower inhibition $(45-70 \%)$ of ERE-ER formation. ${ }^{32}$ P-ERE-ER complex formation was the same in the absence or presence of E2 in 21PT cells, confirming the hormone-independent phenotype of these cells (Figs. 2A \& B, left panels). However, E2 stimulated and W7 inhibited ${ }^{32}$ P-ERE-ER complex 
NMBA
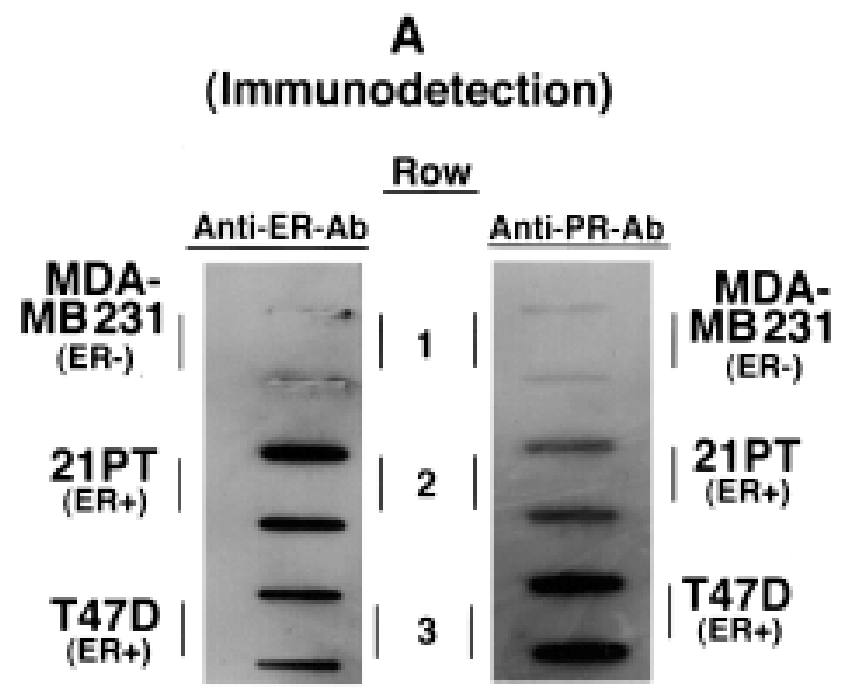

B
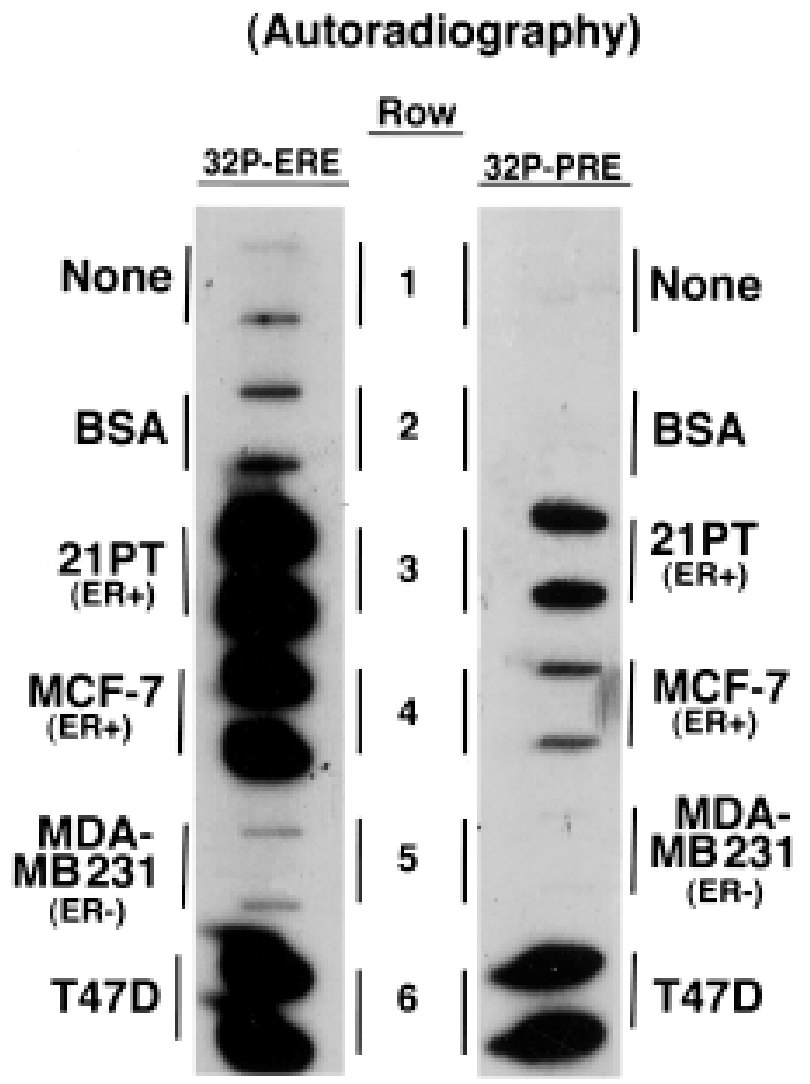

Fig. 1. Nitrocellulose filter binding assay (NMBA) for identification of ERE-ER and PRE-PR complexes. MDA-MB-231, 21PT, MCF-7, and T47D cells were grown in rich medium under standard tissue culture conditions and nuclear extracts were prepared as described by Dignam et al. (25). (A) The total specific receptor (ER) and progesterone receptor (PR) level were measured with NMBA by using the immunodetection assay with nonradioactive estradiol response element (ERE) or progesterone receptor response element (PRE) and (B) the DNA bound formation in the hormone-dependent MCF-7 cells (Figs. 2A \& B, right panels).

A fraction of the reaction mixtures was also analyzed by EMSA. The results presented in Figure 2B demonstrated that ${ }^{32} \mathrm{P}$-ERE-ER complex formation in the extracts of 21PT cells (Fig. 2B, left panel) was inhibited by W7. The E2-induced level of ${ }^{32} \mathrm{P}-\mathrm{ERE}-$ ER complex was less inhibited by W7 in MCF-7 cells (Fig. 2B, right panel), confirming the results with NMBA.

As expected, ER (-) MDA-MB-231 cells were resistant to the antihormone TAM (Fig. 3A). 21PT cells also were resistant to the antihormone TAM, to which ER (+) MCF-7 cells were sensitive (23). However, the antihormone-resistant ER (+) 21PT cells were sensitive to the CaM-antagonist, W7, which correlated with its inhibitory influence on ERE-ER complex formation (Fig. 3B). In contrast the ER (+), MCF-7 cells were sensitive to W7 (Fig. 3B, panel B), and also to TAM (Fig. 3B, panel A), thereby, distinguishing the two ER $(+)$ breast cancer cells on the basis of their sensitivities to these targetdirected drugs. The insensitivity of ER (-) MDAMB-231 cells to W7 suggested that the drug's effect was specific for ER $(+)$ breast cancer cells, both TAM-resistant and -sensitive types. These results, thus, provide a potential therapeutic option for the TAM-resistant 21PT-class of ER (+) human breast cancers by applying inhibitors of the downstream target of ERE-ER interaction.

\section{ER Status in Tumor Biopsy Specimens as Determined by EMSA and NMBA of ${ }^{32} P-E R E-E R$ Interaction}

We subclassified ER (+) and ER (-) human breast cancer frozen biopsy specimens by using a similar experimental approach. Analysis of ER status in 15 human frozen breast tumor biopsy specimens (Table 1) was performed by ${ }^{3} \mathrm{H}-\mathrm{E} 2$ binding (Fig. $4 \mathrm{~A}$ ), by immunodetection (Fig. 4B), and by ${ }^{32} \mathrm{P}-\mathrm{ERE}-$ $E R /{ }^{32}$ P-PRE-PR interaction with NMBA (Fig. 4C). The types, stages, and subclassification of these tumor tissues are described in Table 1.

fraction by using ${ }^{32} \mathrm{P}$-labeled ERE/PRE in the reaction mixture. Immunodetection was carried out by using anti-PR/ER antibody as primary and anti-mouse imunoglobulin G-horse radish peroxide (IgG-HRP) conjugate as secondary, followed by enhanced chemiluminescent protein (ECL) detection analysis as described above. Binding reactions were carried out in a 96-well plate containing $10 \mu \mathrm{g}$ of nuclear protein, double-stranded ${ }^{32} \mathrm{P}$-ERE (1.0 ng and 30,000 cpm) or the same amount of ${ }^{32} \mathrm{P}$-PRE (B) and binding buffer. Following incubation at room temperature for 30 min, the samples were applied to a nitrocellulose membrane in a slot blot assembly system. 15 min later, the membrane was washed three times with binding buffer. Control reactions with no extract (indicated as None, B, row 1) or $10 \mu \mathrm{g}$ of nonspecific protein, such as bovine serum albumin (BSA), were run to determine nonspecific binding of free ${ }^{32} \mathrm{P}$-labeled oligonucleotides to nitrocellulose membrane (B, row 2). A typical result of three such experiments is presented. 

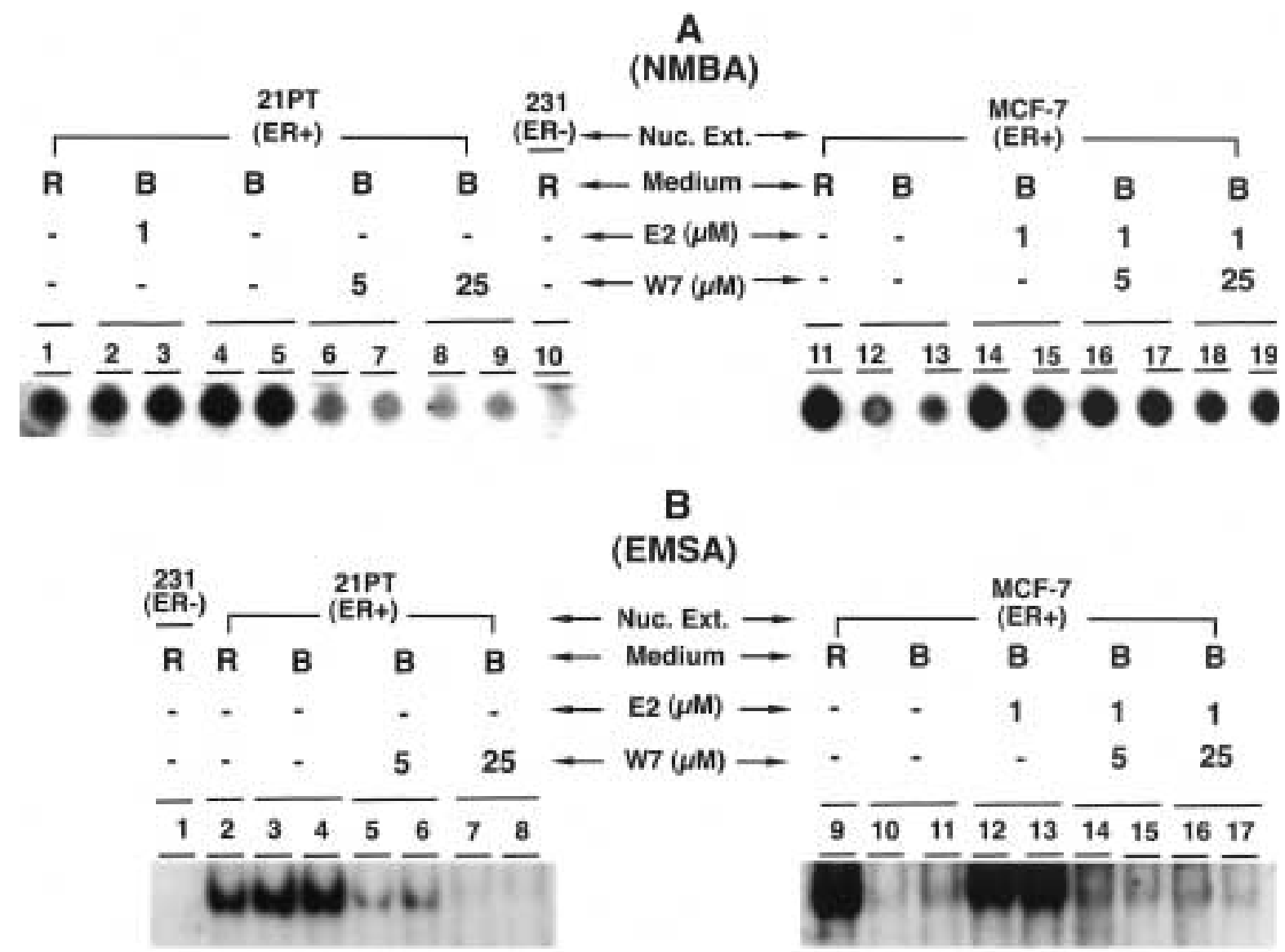

Fig. 2. Effect of CaM-antagonist, W7, on ERE-ER complex formation in ER(+) human breast cancer cell lines. MDA-MB231, $21 \mathrm{PT}$, and MCF-7 cells $\left(10^{5}\right.$ cells $\left./ \mathrm{ml}\right)$ were plated in $25 \mathrm{ml}$ of rich medium in $150 \mathrm{~mm}$ tissue culture dishes. At about $70 \%$ confluency the medium was changed to rich (R) or basal (B) medium [Dulbecco's modified Eagle's medium (DMEM) supplemented with 10\% dextran coated charcoal-treated fetal bovine serum]. $48 \mathrm{hr}$ later the cells were treated with W7, and $1 \mathrm{hr}$ following W7 addition, the cells were treated with estradiol (E2) at the indicated concentrations for an additional $4 \mathrm{hr}$. These cells were harvested and nuclear extracts were made as described (23-25). ${ }^{32} \mathrm{P}$-labeled E2 response element-specific receptor $\left({ }^{32} \mathrm{P}\right.$-ERE-ER) interaction in $10 \mu \mathrm{g}$ of nuclear extracts was measured in duplicate samples by (A) Nitrocellulose filter binding assay (NMBA) and (B) electrophoretic mobility shift assay (EMSA) followed by autoradiography. Control for nonspecific DNA protein interaction was determined by carrying out a reaction with the same amount $(10 \mu \mathrm{g})$ of nuclear extract from ER-deficient breast cancer cell line MDA-MB-231, designated by 231 (panel A, lane 10 and panel B, lane 1). ER+, ER-positive; ER-, ER-negative.

The level of total receptors, ER and PR, and the functional fractions, ${ }^{32} \mathrm{P}-\mathrm{ERE}-\mathrm{ER}$ and ${ }^{32} \mathrm{P}-\mathrm{PRE} / \mathrm{PR}$, were measured in extracts of these tissue speci-mens. ER and PR in 6 out of 15 tissue specimens were positive with regard to all the three criteria and were designated as ER $(+) / P R(+)$ and classified as Class 1 (Fig. 4, Table 1). They showed all the characteristic properties of wild type ER, such as binding with the ligand E2 (Fig. 4A), interaction with anti-ER antibody (Fig. 4B), and binding with ${ }^{32}$ P-ERE (Fig. 4C). As expected, the E2-responsive gene product, PR, also was detected in this class of breast tumors. In contrast, the Class 2 type of ER in 5 of 15 breast tumor tissues did not bind the ligand, could not be detected by the ligand-binding domain-specific antibody, did not interact with ERE, and the E2-responsive gene product PR was not detected. These are designated as ER (-)/PR (-) and are representative of the classical ER (-) mammary epithelial tumor cells, such as MDA-MB-231 and MDA-MB-435. The Class 3 type of ER, identified in two other tumor specimens, bound to E2 and interacted with anti-ERantibody, but did not form ${ }^{32}$ P-ERE-ER complexes. As the E2-responsive gene product PR could be detected in these extracts, E2-ER-mediated action of the hormone appeared to be independent of interaction of the receptor with its response element, ERE, in these tumors. Class 4 of ER, detected in the extracts of two other tissue specimens, in contrast, did not show ligand-binding, but all downstream events, such as ERE-ER interaction and E2-responsive gene (PR) expression, were detected. This class of ER variants had similar properties to those detected in the breast cancer cell line 21PT (22). 

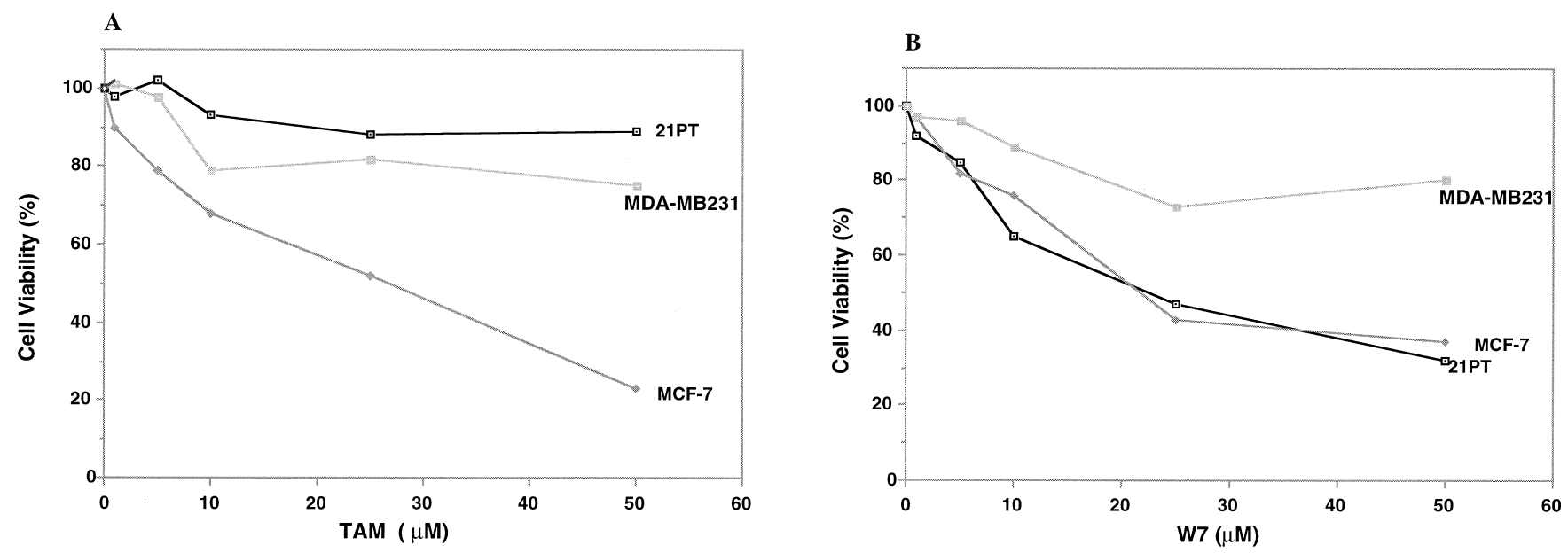

Fig. 3. Sensitivity of the growth of 21PT and MCF-7 cells to (A) tamoxifen (TAM) and (B) W7. MDA-MB231, 21 PT, and MCF-7 cells $\left(2 \times 10^{4}\right)$ were plated in duplicate in 96-well tissue culture dishes in $0.3 \mathrm{ml}$ of rich medium and grown under standard tissue culture conditions. $24 \mathrm{hr}$ later, medium was removed and fresh medium, plus drugs, was added to achieve the indicated concentrations. The cells were grown for an additional 5 days. Cells in control wells were treated with equivalent amounts of solvent (dimethylsulfoxide, DMSO). Medium was changed on alternate days and replaced with fresh medium and drugs. Cell viability in the presence of the drugs was measured by 3-[4,5-dimethylthiazol-2-yl]-2,5-diphenyl tetrazolium bromide (MTT) assay (31) and expressed as percentage of the untreated control. The experiment was repeated three times.

Table 1. Estradiol specific receptor (ER) status in human breast cancer biopsy specimens

\begin{tabular}{|c|c|c|c|c|c|c|c|c|c|}
\hline \multirow[b]{2}{*}{ Tissue \# } & \multirow[b]{2}{*}{ Patient \# } & \multirow[b]{2}{*}{ Class } & \multirow[b]{2}{*}{ Age (yrs) } & \multirow[b]{2}{*}{ Tumor/Stage } & \multirow{2}{*}{$\begin{array}{c}{ }^{3} \mathrm{H}-\mathrm{E} 2 \\
\text { Binding }\end{array}$} & \multicolumn{2}{|c|}{ Immunodetection } & \multicolumn{2}{|c|}{ DNA-Binding } \\
\hline & & & & & & $\mathbf{E R}-\mathbf{A b}$ & PR-Ab & ${ }^{32} \mathrm{P}$-ERE & ${ }^{32}$ P-PRE \\
\hline 1 & 6 & 1 & 51 & Inv. Duct/II & + & + & + & + & + \\
\hline 2 & 7 & 1 & 78 & Inv. Duct/II & + & + & + & + & + \\
\hline 3 & 9 & 1 & 51 & Inv. Duct/II & + & + & + & + & + \\
\hline 4 & 10 & 1 & 41 & Inv. Duct/I & + & + & + & + & + \\
\hline 5 & 12 & 1 & 53 & Inv. Duct/I & + & + & + & + & + \\
\hline 6 & 14 & 1 & 44 & Inv. Lob/I & + & + & + & + & + \\
\hline 7 & 1 & 2 & 58 & DCIS/I & - & - & - & - & - \\
\hline 8 & 2 & 2 & 36 & Inv. Duct/II & - & - & - & - & - \\
\hline 9 & 4 & 2 & 76 & Inv. Duct/II & - & - & - & - & - \\
\hline 10 & 8 & 2 & 48 & Inv. Duct/II & - & - & - & - & - \\
\hline 11 & 15 & 2 & 59 & $\begin{array}{l}\text { Adenocarcinoma } \\
\text { Metastatic/IV }\end{array}$ & - & - & - & - & - \\
\hline 12 & 3 & 3 & 43 & Inv. Duct/I & + & + & + & - & - \\
\hline 13 & 11 & 3 & 72 & Inv. Duct/IV & + & + & + & - & - \\
\hline 14 & 5 & 4 & 80 & Inv. Duct/II & - & + & + & + & + \\
\hline 15 & 13 & 4 & 55 & Inv. Duct/II & - & + & + & + & + \\
\hline \multicolumn{2}{|c|}{ Cell Lines } & Class & & & & & & & \\
\hline \multicolumn{2}{|l|}{ T47D } & 1 & & & + & + & + & + & + \\
\hline \multicolumn{2}{|c|}{ MDA-MB231 } & 2 & & & - & - & - & - & - \\
\hline \multicolumn{2}{|l|}{ None } & 3 & & & & & & & \\
\hline \multicolumn{2}{|l|}{$21 \mathrm{PT}$} & 4 & & & - & + & + & + & + \\
\hline
\end{tabular}

DCIS, ductal carcinoma in situ; Inv. Duct., invasive ductal; Inv. Lob., invasive lobular; +, positive; -, negative; ER(-)Ab, ER(-) antibody; progesterone receptor antibody PR-Ab, ${ }^{32} \mathrm{P}-\mathrm{ERE},{ }^{32} \mathrm{P}$-labeled estradiol response element; ${ }^{32} \mathrm{P}-\mathrm{PRE}$, ${ }^{32} \mathrm{P}-\mathrm{prog}$ esterone receptor response element ${ }^{32} \mathrm{H}-\mathrm{E} 2,{ }^{32} \mathrm{H}$-labeled estradiol. 

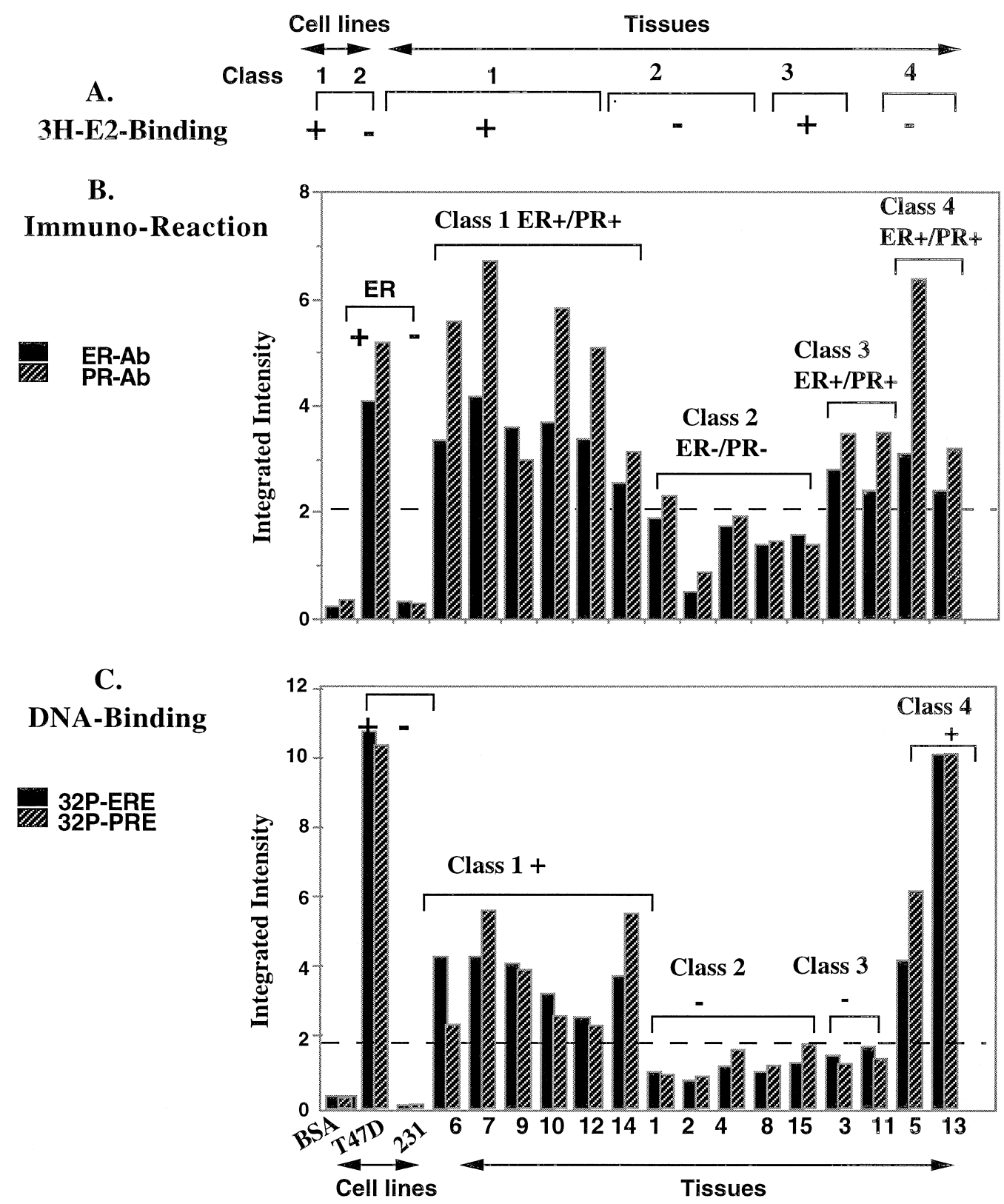

Fig. 4. ER and progesterone receptor (PR) status in breast cancer biopsy specimens. Estradiol specific receptor (ER) and (PR) levels in extracts of frozen human breast tumor biopsy specimens were measured by (A) ${ }^{3} \mathrm{H}-\mathrm{E} 2$ binding using the hydroxylapatite method (32). $10 \mu \mathrm{g}$ (protein) of nuclear extract from cell lines or $20 \mu \mathrm{g}$ of tissue extracts were used for this assay. (B) shows the integrated intensity of the immunoreactive signals generated by the specific interaction of anti-ER or anti-PR antibody with ER or PR. Each reaction of duplicate samples with the immunodetection assay contained $20 \mu \mathrm{g}$ of tissue extracts, 1 ng nonradioactive doublestranded estradiol response element (ERE) oligonucleotide, plus other components, and was incubated under conditions described in "Materials and Methods." Control reactions contained the same amount of bovine serum albumin (BSA) instead of tissue extracts. Nuclear extracts $(10 \mu \mathrm{g})$ from ER-positive [ER (+)] T47D cells and ER-negative [ER (-)] MDA-MB-231 human breast cancer cell lines were analyzed similarly and served as positive and negative controls, respectively. (C) shows the integrated intensity of the autoradiographic signals generated by ${ }^{32} \mathrm{P}$-ERE-ER or ${ }^{32} \mathrm{P}$-PRE-PR complexes immobilized on the nitrocellulose membrane as described in "Materials and Methods." The reactions were run in duplicate under conditions described in the legend to (B), with the exception of the use of double-stranded ${ }^{32} \mathrm{P}$-ERE-oligonucleotide or ${ }^{32} \mathrm{P}-\mathrm{PRE}(1 \mathrm{ng}, 30,000 \mathrm{cpm})$ replacing the nonradioactive oligonucleotides.

Subclassification of these 15 breast tumor tissue specimens on the basis of ER phenotypes (Table 1) demonstrated that ER variants occurred naturally in human breast tumor biopsy specimens. These ER variants were detected on the basis of functional criteria, such as downstream ERE-ER interaction and expression of the hormone responsive gene, PR. They could not be identified by the ligand-binding 
assay or by interaction with a ligand-binding domain-specific anti-ER antibody.

\section{Discussion}

In this study, we identify an alternative target for potential therapy of TAM-resistant ER $(+)$ breast cancers. Initially, we used cell lines as a model working system. Although ER-specific mRNA and protein are detected, the receptor in breast cancer cell line 21PT does not bind to the ligand E2, and so it is TAM-resistant (23). Therefore, we examined a compound targeted at an event downstream of E2ER interaction, the ERE-ER complex formation. Calmodulin (CaM) is an integral component of the ERE-ER complex and is essential for subsequent up-regulation of hormone-responsive promoters (24). Sensitivity to W7 of these TAM-resistant breast cancer cells can be explained on the basis of specific binding with $\mathrm{CaM}$, thereby, preventing participation of CaM in ERE-ER complex formation (24).

We established and used a simplified assay system for identification of DNA-protein complexes by a procedure (NMBA) that is not as complicated and that does not involve as many steps as the classical DNA-protein interaction assay, EMSA (20).

Analysis of 15 breast tumor biopsy specimens revealed that, similar to 21PT, ER in two of these tumor cells interacted with the ERE sequence independent of binding of the hormone. These tumors, similar to 21PT cells, should be responsive to W7 or to other compounds that block ERE-ER interaction. This strategy of identifying ER variants would overcome the lack of a target-directed therapy for ER (+) antihormone-resistant breast cancer patients. It is dependent on the determination of the level and functional state of ER.

We identify four different classes of breast cancers, among the few biopsy specimens examined in this study, on the basis of functional state of ER (Table 1). The Class 1 human breast cancers designated as ER $(+) / P R(+)$, should be responsive to antihormone therapy. In contrast, ER can not be detected in Class 2 breast tumors by any one of these parameters and, thus, are classified as ER $(-) / P R(-)$. These breast cancer patients should not respond to antihormone therapy. The Class 3 breast cancers are ER $(+)$ on the basis of ligand binding, but ERE-ER interaction was not detected and, thus, these patients also should not respond to antihormones. The hormone-induced regulation of gene expression apparently is mediated via a mechanism independent of ERE-ER interaction, possibly via protein-protein interaction $(27,28)$. Finally, the ER in Class 4 breast cancer biopsy specimens did not show E2 binding, but the downstream functions were evident. These breast cancers, comparable to 21PT cells, should be resistant to antihormones. But, they should be sensitive to the CaM-antagonist W7.
Treatment with antihormones targeted at ER is a current therapy for breast cancer patients. The critical issue still remains to be resolved: what targetdirected therapies could be effective for the numerous antihormone-resistant ER (+) and ER (-) breast cancer patients? These patients currently are subjected to random combination chemotherapy. Treatment with Herceptin, an antibody to the EGFR family of receptors also is used with limited success for breast cancers overexpressing these receptors (29). Our recent results predict that NF- $\kappa$ B could be a potential target for therapy of ER (-) breast cancer patients with inhibtors of this nuclear transcription factor (18). Another proposed new approach is antivascular therapy (30). Results presented demonstrate that the strategy applied in this study for analyzing functional status of ER in human breast tumor biopsy specimens could be useful for making therapeutic predictions for patients with different classes of breast cancers.

\section{Acknowledgments}

The work was supported by funds from the Commonwealth of Massachusetts, Department of Public Health and Breast Cancer Research Grants Program and National Institutes of Health NCI Grant CA61253-07.

\section{References}

1. Jensen EV. (1981) Hormone dependency in breast cancer. Breast Cancer Res. Treat. 26: 2319-2326.

2. Jordan VC. (1992) The strategic use of antiestrogens to control the development and growth of breast cancer. Cancer 70: 977-982.

3. Hortobagy GN. (1998) Treatment of breast cancer. N. Eng. J. Med. 339: 974-984.

4. Lazennec G, Katzenellenbogen BS. (1999) Expression of human estrogen receptor using an efficient adenoviral gene delivery system is able to restore hormone dependent features to estrogen receptor-negative breast carcinoma cells. Mol. Cell Endocrionol. 149: 93-105.

5. Lazennec G, Alcorn JL, Katzenellenbogen BS. (1999) Adenovirus-mediated delivery of dominant negative estrogen receptor gene abrogates estrogen stimulated gene expression and breast cancer cell proliferation. Mol. Endocrinol. 13: 969-980.

6. Smith DF, Toft DO. (1993) Steroid receptors and their associated proteins. Mol. Endocrinol. 7: 4-11.

7. Kumar V, Chambon P. (1988) The estrogen receptor binds tightly to its response element as a ligand-induced homodimer. Cell 55: 145-156.

8. Tsai S-Y, Tsai M-J., O'Malley BW. (1989) Cooperative binding of steroid hormone receptors contributes to transcriptional synergism at target enhancer elements. Cell 57: 443-448.

9. Brown M. (1994) Estrogen receptor molecular biology. Hemat. Oncol. Clin. N. Amer. 8: 101-112.

10. Jordan VC. (1995) Studies on the estrogen receptor in breast cancer, 20 years as target for the treatment and prevention of cancer. Breast Cancer Res. Treat. 36: 267-285.

11. Shibata H, Spencer TE, Sergio AO, et al. (1997) Role of coactivators and co-repressors in the mechanism of steroid/ thyroid receptor action. Recent Prog. Hormone Res. 52: 141-165.

12. Smith CL, Conneely M, O'Malley BW. (1993) Modulation of the ligand-independent activation of the human estrogen receptor by hormone and antihormone. Proc. Natl. Acad. Sci. USA 90: 6120-24. 
13. Osborne CK. (1991) Receptors In Breast Diseases, In Breast Cancer and Diseases. Harris JR, Hellerman S, Henderson IC, Kinne DW (eds.). 2nd Edition, Lippincott, Philadelphia, pp. 301-325.

14. Hedden A, Muller V, Jensen EV. (1995) A new interpretation of antiestrogen action. NY Acad. Sci. 761: 109-120.

15. Paige LA, Christensen DJ, Gron H, et al. (1999) Estrogen receptor (ER) modulators each induce distinct conformational changes in ER $\alpha$ and ER $\beta$. Proc. Natl. Acad. Sci. USA 96: 3999-4004.

16. Love RR, Mazess RB, Barden HS, et al. (1992) Effects of tamoxifen on bone mineral density in postmenopausal women with breast cancer. N. Engl. J. Med. 326: 852-856.

17. Kedar RP, Bourne TH, Powles TJ, et al. (1994) Effects of tamoxifen on uterus and ovaries of postmenopausal women in a randomized breast cancer prevention trial. Lancet 343: 1318-1321.

18. Biswas DK, Cruz AP, Gansberger E, Pardee AB. (2000) Epidermal growth factor-induced nuclear factor $\kappa \mathrm{B}$ activation: a major pathway of cell cycle progression in estrogen-receptor negative breast cancer cells. Proc. Natl. Acad. Sci. USA 97: 8542-8547.

19. Skaar TC, Prasad SC, Sharareh S, Lippman ME, Brunner N, Clarke R. (1998) Two dimensional gel electrophoresis analysis identify nucleoplasmin as an estrogen regulated protein associated with acquired estrogen-independence in human breast cancer cells. J. Steroid Chem. 67: 391-402.

20. Biswas DK, Cruz A, Pardee AB. (2000) Detection of estrogen receptor functional variants in human breast cancers by a novel approach. Biotechniques (In Press).

21. Sager R, Sheng S, Anisowicz A, et al. (1994) RNA genetics of breast cancer: maspin as paradigm. Cold Spring Harbor Symp. Quant. Biol. LIX: 537-546

22. Zajchowski DA, Band V, Trask DK, Kling D, Connolly, JL, Sager R. (1990) Suppression of tumor-forming ability and related traits in MCF-7 human breast cancer cells by fusion with mammary epithelial cells. Proc. Natl. Acad. Sci. USA 87: 2314-2318.

23. Biswas DK, Averboukh L, Sheng S, et al. (1998) Classification of breast cancer cells on the basis of a functional assay for estrogen receptor. Mol. Med. 4: 454-467.

24. Biswas DK, Reddy PV, Pickard M, Makkad B, Pettit N, Pardee AB. (1998) Calmodulin is essential for estrogen receptor interaction with its motif and activation of responsive promoter. J. Biol. Chem. 273: 33817-33824.

25. Dignam JD, Lebovitz RM, Roeder RD. (1983) Accurate transcription initiation by RNA polymerase II in a soluble extract from isolated mammalian nuclei. Nucleic Acids Res. 11: 14751489.

26. Bradford MM. (1978) A rapid sensitive method for the quantitation of microgram quantities of protein utilizing the principle of protein dye binding. Anal. Biochem. 72: 248254.

27. Porter W, Wang F, Wang W, Duan R, Safe S. (1996) Role of estrogen receptor/Spl complexes in estrogen-induced heat shock protein 27 gene expression. Mol. Endocrinol. 11: 13711378.

28. Sukovich DA, Kukherjee R, Benfield, PA. (1994) A novel celltype specific mechanism for estrogen receptor mediated gene activation in the absence of estrogen-responsive element. Mol. Cell Biol. 14: 7134-7143.

29. Pegram MD, Pauletti G, Slamon DJ. (1998) HER2/neu as a predictive marker of response to breast cancer therapy. Breast Cancer Res. Treat. 52: 155-167.

30. Hayes AJ, Li LY, Lippman ME. (1999) Science, medicine and the future. Brit. Med. 318: 853-856.

31. Mosman T. (1983) Rapid colorimetric assay for cellular growth and survival: application to proliferation and cytotoxic assay. J. Immunol. Meth. 65: 55-64.

32. Obourn JD, Koszewski NJ, Notides AC. (1993) Hormoneand DNA binding mechanisms of the recombinant human estrogen receptor. Biochemistry 32: 6229-6236. 